\title{
EFEITOS DA INOCULAÇÃO COM RIZÓBIO E DA ADUBAÇÃO NITROGENADA NA PRODUÇÃO DE MUDAS DE SESBÂNIA EM SUBSTRATO CONSTITUÍDO DE RESÍDUOS AGROINDUSTRIAIS ${ }^{1}$
}

\author{
Luciana de Lima Brandão Chaves² ${ }^{2}$ José Geraldo de Araújo Carneiro ${ }^{3}$, Deborah Guerra Barroso ${ }^{3}$ e \\ Paulo Sérgio dos Santos Leles ${ }^{4}$
}

\begin{abstract}
RESUMO - Com o objetivo de avaliar o efeito de diferentes fontes e doses de $\mathrm{N}$ e da inoculação com rizóbio na produção de mudas de Sesbania virgata (sesbânia), conduziu-se um experimento no qual se utilizou como substrato composto de bagaço de cana-de-açúcar + torta de filtro de usina peneirados (3:2, v:v) e decompostos. Foram testados os seguintes tratamentos em esquema fatorial: 3 doses de $\mathrm{N}\left(100,200\right.$ e $\left.300 \mathrm{mg} / \mathrm{dm}^{3}\right) \times 3$ doses de $\mathrm{N}$ (uréia, sulfato de amônio e nitrato de amônio) + 2 tratamentos adicionais (inoculado e não-inoculado com rizóbio). As três adubações foram realizadas a cada 15 dias, sendo a primeira 30 dias após a semeadura. $\mathrm{O}$ experimento foi conduzido sob delineamento inteiramente casualizado, com três repetições compostas por 80 mudas. As mudas foram avaliadas em altura, diâmetro, massas de matéria seca da parte aérea e do sistema radicular, área foliar e potencial de regeneração de raízes. Conclui-se que para este substrato a inoculação das sementes foi suficiente para atender à demanda de $\mathrm{N}$ das mudas de sesbânia.
\end{abstract}

Palavras-chave: Sesbânia, fertilização e produção de mudas.

\section{EFFECT OF RHIZOBIUM INOCULATION AND NITROGEN APPLICATIONS ON SESBÂNIA SEEDLINGS PRODUCED IN SUBSTRATES COMPOSED OF AGRO-INDUSTRIAL RESIDUES}

\begin{abstract}
An experiment was carried out to evaluate the effect of different sources and doses of nitrogen and Rhizobium inoculation on the production of sesbânia seedlings (Sesbania virgata). The substrate was decomposed sugarcane bagasse + filter cake (3:2; v:v). The statistical design was a $3 \times 3 \times 2$ factorial scheme ( $3 \mathrm{~N}$ doses - 100, 200 and $300 \mathrm{mg} / \mathrm{dm}^{3} \times 3 \mathrm{~N}$ sources - urea, ammonium sulfate and ammonium nitrate + two additional treatments with and without Rhizobium inoculation. The experiment was arranged in a randomized complete design, with three replicates. Each experimental plot was composed of 80 seedlings. Height, diameter, shoot and root dry matter weights, leaf area and root regeneration potential of the seedlings were evaluated. It was concluded that, for this substrate, sesbânia seedlings can be produced without the addition of nitrogen fertilizers.
\end{abstract}

Key words: Sesbânia, fertilization, seedlings.

\section{INTRODUÇÃO}

A Sesbania virgata, pertencente à família Leguminosae, é uma espécie pioneira, arbustiva e semi-perene que forma simbiose radicular com Azorhizobium spp. (Santos et al., 1998). Esta espécie apresenta alto potencial para utilização em programas de recuperação de áreas degradadas.

1 Recebido para publicação em 1.10.2001. Aceito para publicação em 9.9.2003.

2 Enga. Florestal, M.S. em Produção Vegetal, LFIT/CCTA da Universidade Estadual do Norte Fluminense - UENF.; ${ }^{3}$ Prof. do Setor de Silvicultura, CCTA/UENF, <carneiro@uenf.br>; <deborah@uenf.br>. ${ }^{4}$ Prof. Silvicultura, UFRRJ, <pleles@ufrrj.br>. 
As espécies da família Leguminosae têm a capacidade de fixar biologicamente o $\mathrm{N}$ e as bactérias podem fornecer à planta de 60 a $100 \%$ de suas necessidades de N (Costa Júnior, 1997). Há uma especificidade entre a bactéria e a planta, segundo Freire (1992).

Segundo Faria (1998), a Sesbania virgata demonstrou ser bastante específica, apresentando nodulação eficiente somente com estirpes isoladas dela própria ou do mesmo gênero.

Entre os diversos resíduos agroindustriais utilizados na produção de mudas na região Norte-Fluminense, destacam-se o bagaço de cana-de-açúcar e a torta de filtro de usina açucareira, que são descartados, podendo gerar impactos negativos ao meio ambiente. Desta forma, Morgado (1998) concluiu que a mistura de bagaço de cana-de-açúcar e torta de filtro de usina açucareira (3:2, v:v) adequou-se à produção de mudas de Eucalyptus grandis e Saccharum officinarum. Com este mesmo substrato, Samôr (1999) produziu com êxito mudas de Anadenanthera macrocarpa e Sesbania virgata.

Entretanto, estudando o comportamento de mudas de sabiá (Mimosa caesalpiniifolia) e aroeira (Schinus terebinhtifolius), produzidas neste substrato, com diferentes doses de nitrogênio, potássio, enxofre e micronutrientes, Barroso et al. (1998) concluíram que o nitrogênio foi o único nutriente limitante na utilização deste substrato para produção das espécies estudadas.

Para fornecer condições ao desenvolvimento das mudas nesse substrato, pode ser necessária a adubação nitrogenada. Como fonte de $\mathrm{N}$, podem ser utilizados a uréia, o sulfato de amônio e o nitrato de amônio.

Portanto, neste trabalho objetivou-se avaliar o efeito de diferentes fontes e doses de adubos nitrogenados e da inoculação com rizóbio na produção de mudas de Sesbania virgata (sesbânia), em substrato constituído por composto de resíduos agroindustriais.

\section{MATERIAL E MÉTODOS}

O trabalho foi conduzido na Estação Experimental da PESAGRO - RIO, em Campos dos Goytacazes-RJ.

A produção das mudas obedeceu às seguintes etapas: semeadura em casa de vegetação (45 dias), transferência para área sombreada, com utilização de sombrite (50\%) na parte superior (10 dias) e rustificação a pleno sol (20 dias).

A sesbânia teve seus frutos coletados e secos, e suas sementes foram extraídas manualmente após abertura do legume. Foi realizada quebra de dormência das sementes com água aquecida a $70^{\circ} \mathrm{C}$ (Samôr, 1999) + escarificação mecânica e, de acordo com os tratamentos utilizados, elas foram inoculadas com rizóbio específico para Sesbania virgata - estirpe BR 5401 e BR 5412, obtido no CNPAB-EMBRAPA - Seropédica. Foram utilizadas duas sementes por recipiente, para posterior repicagem.

As mudas de sesbânia foram produzidas em sacos de polietilino preto $(18 \times 15 \mathrm{~cm})$, contendo substrato constituído por bagaço de cana-de-açúcar e torta de filtro de usina açucareira (3:2, v:v), que foram individualmente secos e peneirados (malha de $0,5 \mathrm{~cm}$ ). Após a mistura, foi feita a compostagem do material, visando a diminuição da relação $\mathrm{C} / \mathrm{N}$, tendo sido adicionados $500 \mathrm{~g}$ de uréia/1.500 1 de substrato para acelerar o processo de compostagem. Durante esta etapa, o material foi revolvido e umedecido a cada semana.

Posteriormente, esse substrato foi tratado com brometo de metila, na dosagem de $60 \mathrm{ml} / 1.5001$ de substrato.

A composição química desse substrato, obtida através de digestão sulfúrica e nitroperclórica, encontra-se no Quadro 1 e foi realizada no Laboratório da Escola Técnica Federal de Campos dos Goytacazes.

Quadro 1 - Análise química do substrato: composto de bagaço de cana-de-açúcar + torta de filtro (3:2, v:v) Table 1 - Chemical analysis of the substrate: sugarcane bagasse + filter cake $(3: 2, v: v)$

\begin{tabular}{|c|c|c|c|c|c|c|c|c|c|c|}
\hline $\mathrm{U}$ & $\mathrm{N}$ & $\mathrm{P}_{2} \mathrm{O}_{5}$ & $\mathrm{~K}_{2} \mathrm{O}$ & $\mathrm{C}$ & $\mathrm{Ca}$ & $\mathrm{Mg}$ & $\mathrm{Fe}$ & $\mathrm{Cu}$ & $\mathrm{Zn}$ & $\mathrm{Mn}$ \\
\hline$(\%)$ & $\left(\mathrm{g} / \mathrm{dm}^{3}\right)$ & \multicolumn{2}{|c|}{$\left(\mathrm{mg} / \mathrm{dm}^{3}\right)$} & $\left(\mathrm{g} / \mathrm{dm}^{3}\right)$ & \multicolumn{4}{|c|}{$\left(\mathrm{mmol}_{\mathrm{c}} / \mathrm{dm}^{3}\right)$} & \multicolumn{5}{|c|}{$\left(\mathrm{mg}^{3} \mathrm{dm}^{3}\right)$} \\
\hline 53 & 8,5 & 19,5 & 1,6 & 76,8 & 101,5 & 13,33 & 10560 & 32 & 114 & 500 \\
\hline
\end{tabular}


Os tratamentos utilizados foram: T1: sem inoculação - N; T2: com inoculação $-\mathrm{N}$; T3: sem inoculação +100 $\mathrm{mg} / \mathrm{dm}^{3}$ de $\mathrm{N}$ (uréia); T4: sem inoculação $+200 \mathrm{mg} / \mathrm{dm}^{3}$ de $\mathrm{N}$ (uréia); T5: sem inoculação $+300 \mathrm{mg} / \mathrm{dm}^{3}$ de $\mathrm{N}$ (uréia); T6: sem inoculação $+100 \mathrm{mg} / \mathrm{dm}^{3}$ de $\mathrm{N}$ (sulfato de amônio); T7: sem inoculação $+200 \mathrm{mg} / \mathrm{dm}^{3} \mathrm{de}$ $\mathrm{N}$ (sulfato de amônio); T8: sem inoculação $+300 \mathrm{mg} /$ $\mathrm{dm}^{3}$ de $\mathrm{N}$ (sulfato de amônio); T9: sem inoculação + $100 \mathrm{mg} / \mathrm{dm}^{3}$ de $\mathrm{N}$ (nitrato de amônio); T10: sem inoculação $+200 \mathrm{mg} / \mathrm{dm}^{3}$ de $\mathrm{N}$ (nitrato de amônio) e T11: sem inoculação $+300 \mathrm{mg} / \mathrm{dm}^{3}$ de $\mathrm{N}$ (nitrato de amônio).

$\mathrm{Na}$ etapa de produção de mudas, usou-se o delineamento inteiramente casualizado, com dois tratamentos adicionais (T1 e T2) e um arranjo fatorial 3x3 (três fontes de $\mathrm{N}$ e três doses de $\mathrm{N}$ ), com três repetições, compostas por 80 mudas cada uma.

A aplicação dos fertilizantes às mudas foi parcelada em três vezes, com intervalos de 15 dias, sendo a primeira aplicação realizada 30 dias após a semeadura.

A seleção das mudas para avaliação foi feita de maneira sistemática, medindo-se plantas intercaladas, respeitando-se a bordadura de uma linha simples de mudas.

Aos 75 dias após a semeadura as mudas foram avaliadas em altura (H); diâmetro do colo (D); relação H/D; massa de matéria seca da parte aérea (PSA), do sistema radicular (PSR), total (PST) e PSR/PSA; área foliar (AF), e submetidas a testes para avaliação do potencial de regeneração de raízes (PRR).

A área foliar foi avaliada por meio de medidor de área eletrônico de bancada (LI-3000, LI-COR Inc.). Em seguida, as mudas foram seccionadas em parte aérea e radicular e colocadas em estufa de circulação forçada a $75^{\circ} \mathrm{C}$, até atingirem massa constante. Após a secagem, foi determinada a massa de matéria seca da parte aérea (PSA) e do sistema radicular (PSR).

Na avaliação do PRR, o delineamento usado foi o inteiramente casualizado, com dois tratamentos adicionais e um arranjo fatorial $3 \times 3$, com cinco repetições, sendo cada repetição composta por uma muda.

Para o PRR, usaram-se dois tipos de rizotrons: aquários e tubos plásticos transparentes, segundo metodologia usada por Carneiro (1995), Novaes (1998), Morgado (1998), Leles (1998), Barroso (1999) e Samôr (1999). As mudas selecionadas para esta avaliação apresentavam dimensões de altura e diâmetro mais próximas da média calculada em cada tratamento.

Nos aquários foi usada solução nutritiva de Bolles Jones, à meia-força, e durante o ensaio (15 dias) não foi detectada variação acentuada do $\mathrm{pH}$, permanecendo em torno de 6,0 .

Após 15 dias, as mudas foram retiradas dos aquários e as raízes regeneradas, a partir dos pontos de poda, foram avaliadas em número e comprimento.

Nos tubos, a cada três dias (quatro contagens), foram feitas contagens do número de extremidades e comprimento de raízes regeneradas, visíveis em toda a superfície interna das paredes dos tubos. O substrato usado foi areia e estéril de extração de argila (3:2, v:v). A análise química deste substrato encontra-se no Quadro 2.

Os dados de todos os parâmetros foram submetidos à análise de variância e os tratamentos foram comparados por contrastes e teste de Scheffé $(\mathrm{P}<0,05)$. $\mathrm{O}$ primeiro contraste foi entre o grupo dos tratamentos adicionais

Quadro 2 - Análise química do substrato (areia+ substrato de cava de extração de argila, 3:2, v:v)

Table 2 - Chemical analysis of substrate (sand + substratum of clay mining, 3:2, v:v)

\begin{tabular}{|c|c|c|c|c|c|c|c|c|c|c|}
\hline $\mathrm{pH}$ & $\mathrm{P}^{1 /}$ & $\mathrm{K}^{1 /}$ & $\mathrm{Ca}$ & $\mathrm{Mg}$ & $\mathrm{Al}$ & $\mathrm{H}+\mathrm{Al}$ & $\mathrm{Fe}$ & $\mathrm{Cu}$ & $\mathrm{Zn}$ & Mn \\
\hline & \multicolumn{2}{|c|}{$\left(\mathrm{mg} / \mathrm{dm}^{3}\right)$} & \multicolumn{4}{|c|}{$\left(\mathrm{cmol}_{\mathrm{c}} / \mathrm{dm}^{3}\right)$} & \multicolumn{4}{|c|}{$\left(\mathrm{mg} / \mathrm{dm}^{3}\right)$} \\
\hline 6,7 & 34 & 22 & 1,5 & 0 & 0,0 & 1,2 & 30,0 & 0,2 & 3,6 & 10,0 \\
\hline \multicolumn{2}{|c|}{$\mathrm{C}$} & \multicolumn{2}{|c|}{ MO } & S.B. & \multicolumn{2}{|c|}{$\mathrm{T}$} & $\mathrm{t}$ & \multicolumn{2}{|c|}{$\mathrm{m}$} & $\mathrm{V}$ \\
\hline \multicolumn{2}{|c|}{$(\%)$} & \multicolumn{2}{|c|}{$\left(\mathrm{g} / \mathrm{dm}^{3}\right)$} & \multicolumn{4}{|c|}{$\left(\mathrm{cmol}_{\mathrm{c}} / \mathrm{dm}^{3}\right)$} & \multicolumn{3}{|c|}{$(\%)$} \\
\hline \multicolumn{2}{|c|}{0,41} & \multicolumn{2}{|c|}{7,1} & 2,0 & \multicolumn{2}{|c|}{3,2} & 2,0 & \multicolumn{2}{|c|}{0} & 62 \\
\hline
\end{tabular}

${ }^{1 /}$ Extrator Carolina do Norte. 
(G1) e o grupo dos tratamentos do arranjo fatorial (G2). $\mathrm{O}$ segundo contraste foi entre os dois tratamentos adicionais (T1 e T2). Já o contraste seguinte testou o tratamento adicional sem inoculação e sem adubação (T1 controle) e os tratamentos do arranjo fatorial. Por último, comparou-se o tratamento adicional, no qual as sementes foram inoculadas e as mudas não receberam a aplicação de N (T2 - inoculado), com os tratamentos do arranjo fatorial $(\mathrm{G} 2)$.

Foram realizadas análises de regressão para os fatores quantitativos significativos e para os fatores qualitativos, contrastes e teste de Scheffé, comparando-se a fonte 1 (uréia) com o grupo formado pelas fontes 2 (sulfato de amônio) e 3 (nitrato de amônio), e ainda testou-se a fonte 1 contra a fonte 2 .

\section{RESULTADOS E DISCUSSÃO}

De acordo com as análises de variância, constatouse que entre as características morfofisiológicas avaliadas houve diferenças entre as fontes de $\mathrm{N}$ aplicadas sobre a altura (H), diâmetro do colo (D) e no PRR, com exceção do comprimento total das raízes avaliado em solução nutritiva.

Também foi observado efeito das doses dos nutrientes no comprimento médio das raízes avaliado em solução nutritiva e nas avaliações de PRR realizadas nos tubos.

Embora tenham sido encontradas diferenças nas análises de variância, elas não foram detectadas através dos contrastes (Quadros 3, 4 e 5).

A primeira comparação foi feita entre o grupo dos tratamentos adicionais (G1) e o grupo dos tratamentos do arranjo fatorial (G2), para todas as características
Quadro 3 - Contrastes realizados entre os tratamentos das mudas de Sesbania virgata, na avaliação de altura $(\mathrm{H})$, diâmetro do colo (D), número de raízes $(\mathrm{N})$ e comprimento médio de raízes regeneradas (CM)

Table 3 - Contrasts made among the treatments in Sesbania virgata seedlings to evaluate height $(H)$, collar diameter $(D)$, number $(N)$ and medium length of regenerated roots (CM)

\begin{tabular}{|c|c|c|c|c|}
\hline \multirow[t]{2}{*}{ Tratamento } & \multicolumn{2}{|c|}{$\begin{array}{c}\text { Característica } \\
\text { Morfológica }\end{array}$} & \multicolumn{2}{|c|}{$\begin{array}{c}\text { PRR (solução } \\
\text { hidropônica) }\end{array}$} \\
\hline & $\mathrm{H}$ & $\mathrm{D}$ & $\mathrm{N}^{2 /}$ & $\mathrm{CM}$ \\
\hline $\mathrm{T} 1$ e T2 $\times$ G $2^{\frac{1}{\prime}}$ & $117,4139^{\text {ns }}$ & $1,2385^{\mathrm{ns}}$ & $3,9662^{\text {ns }}$ & $49,8518^{n}$ \\
\hline $\mathrm{T} 1 \times \mathrm{T} 2$ & $11,8006^{\mathrm{ns}}$ & $1,0117^{\text {ns }}$ & $0,3986^{\mathrm{ns}}$ & $5,0103^{\mathrm{n}}$ \\
\hline $\mathrm{T} 1 \times \mathrm{T} 3$ & $79,1604^{\mathrm{ns}}$ & $6,7835^{\text {ns }}$ & $2,6741^{\text {ns }}$ & $33,6101^{\mathrm{n}}$ \\
\hline $\mathrm{T} 2 \times \mathrm{T} 3$ & $79,1604^{\mathrm{ns}}$ & $6,7835^{\text {ns }}$ & $2,6741^{\mathrm{ns}}$ & $33,6101^{\mathrm{n}}$ \\
\hline
\end{tabular}

1/ G2: grupo formado pelos tratamentos do arranjo fatorial, $2 /$ dados transformados em $\log (\mathrm{X}+10) \mathrm{e}^{\mathrm{ns}}$ não-significativo pelo teste de Scheffé $(\mathrm{P}>0,05)$.

Quadro 4 - Contrastes realizados entre os tratamentos das mudas de Sesbania virgata, na avaliação número de extremidade de raízes regeneradas (N) e do comprimento de raízes regeneradas $(\mathrm{C})$

Table 4 - Contrasts made among the treatments in Sesbania virgata seedlings, treatments to evaluate number of top $(N)$ and length $(C)$ of regenerated roots

\begin{tabular}{|c|c|c|}
\hline \multirow{2}{*}{ Tratamento } & \multicolumn{2}{|c|}{ PRR (tubos) } \\
\hline & $\mathrm{N}^{2 / 1}$ & $\mathrm{C}$ \\
\hline $\mathrm{T} 1$ e T $2 \times \mathrm{G} 2^{1 / 1}$ & $4,5605^{\mathrm{ns}}$ & $4,2564^{\mathrm{n}}$ \\
\hline $\mathrm{T} 1 \times \mathrm{T} 2$ & $0,4587^{\mathrm{ns}}$ & $0,4278^{\mathrm{n}}$ \\
\hline $\mathrm{T} 1 \times \mathrm{T} 3$ & $3,0747^{\mathrm{ns}}$ & $2,8697^{\mathrm{n}}$ \\
\hline $\mathrm{T} 2 \times \mathrm{T} 3$ & $3,0747^{\text {ns }}$ & $2,8697^{\mathrm{n}}$ \\
\hline
\end{tabular}

1/ G2: grupo formado pelos tratamentos do arranjo fatorial, 2/ dados transformados em $\log (\mathrm{X}+10) \mathrm{e}^{\mathrm{ns}}$ não-significativo pelo teste de Scheffé $(\mathrm{P}>0,05)$.

Quadro 5 - Contrastes realizados entre os tratamentos das fontes significativas das mudas de Sesbania virgata, na avaliação de altura $(\mathrm{H})$, diâmetro do colo $(\mathrm{D})$, número de raízes $(\mathrm{N})$ e comprimento médio de raízes regeneradas $(\mathrm{CM})$

Table 5 - Contrasts among the treatments of significant nitrogen source, of Sesbania virgata seedlings to evaluate height $(H)$, collar diameter $(D)$, number $(N)$ and medium length $(C M)$ of regenerated roots

\begin{tabular}{|c|c|c|c|c|c|c|}
\hline \multirow{2}{*}{ Tratamento $^{1 /}$} & \multicolumn{2}{|c|}{ Características Morfológicas } & \multicolumn{2}{|c|}{ PRR (solução hidropônica) } & \multicolumn{2}{|c|}{ PRR (tubos) } \\
\hline & $\mathrm{H}$ & $\mathrm{D}$ & $\mathrm{N}^{2} /$ & $\mathrm{CM}$ & $\mathrm{N}^{2} /$ & $C^{2} /$ \\
\hline $\mathrm{F} 1 \times \mathrm{F} 2$ e F3 & $35,40^{\mathrm{ns}}$ & $3,03^{\text {ns }}$ & $1,49^{\text {ns }}$ & $18,73^{\text {ns }}$ & $1,76^{\mathrm{ns}}$ & $1,64^{\mathrm{ns}}$ \\
\hline $\mathrm{F} 1 \times \mathrm{F} 2$ & $20,43^{\mathrm{ns}}$ & $1,75^{\mathrm{ns}}$ & $0,86^{\mathrm{ns}}$ & $10,81^{\mathrm{ns}}$ & $1,01^{\mathrm{ns}}$ & $0,86^{\mathrm{ns}}$ \\
\hline
\end{tabular}

${ }^{1 /} \mathrm{F} 1$ : fonte - uréia $\left(100,200\right.$ e $\left.300 \mathrm{mg} / \mathrm{dm}^{3}\right)$, F2: fonte - sulfato de amônio $\left(100,200\right.$ e $\left.300 \mathrm{mg} / \mathrm{dm}^{3}\right)$ e F3: fonte - nitrato de amônio (100,

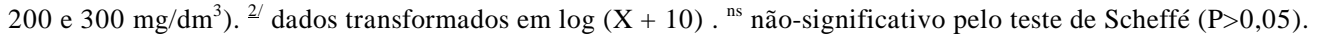


avaliadas, obtendo-se contrastes não-significativos. Tal resultado indica que as médias dos tratamentos adicionais são estatisticamente equivalentes às médias dos tratamentos que receberam adubação nitrogenada. Sendo assim, pode-se inferir que não houve efeito da aplicação do nitrogênio sobre as mudas de sesbânia.

Ao comparar as características entre os dois tratamentos adicionais, verifica-se também que os contrastes não foram significativos. Neste caso, a inoculação das sementes com o rizóbio específico não possibilitou incremento em altura e diâmetro das mudas, provavelmente em função de uma nodulação natural eficiente apresentada pela espécie nas condições do experimento.

A comparação das características por contrastes, entre o tratamento adicional sem inoculação e sem adubação (controle) e os tratamentos do arranjo fatorial, não apresentou diferenças. Conclui-se que para a espécie em questão a aplicação de diferentes doses de N, em cada fonte de adubo nitrogenado usado, não alterou o crescimento em altura e diâmetro das mudas.

O mesmo foi observado ao comparar o tratamento adicional, no qual as sementes foram inoculadas e as mudas não receberam a aplicação de N (inoculado), com os tratamentos do arranjo fatorial, que receberam adubação nitrogenada. Os contrastes também não apresentaram diferenças, o que reforça o fato de a adubação nitrogenada não ser necessária para a espécie em questão, quando produzida nesse substrato.
Também não foram detectadas, por contrastes, diferenças entre as fontes de $\mathrm{N}$ usadas nas características morfofisiológicos avaliadas.

Barroso et al. (2000), trabalhando com o mesmo substrato e a mesma espécie, encontraram resultados diferentes, pois o aumento das doses de $\mathrm{N}$ possibilitou maior incremento em altura, diâmetro, área foliar e matéria seca da parte aérea e do sistema radicular, chegando à determinação de que a dose recomendada para a Sesbania virgata é de $450 \mathrm{mg} / \mathrm{kg}$ de N. Os autores sugerem que a resposta das mudas ao $\mathrm{N}$ aplicado é devido a uma possível baixa eficiência de nodulação natural, uma vez que não houve inoculação das sementes, o que poderia explicar a diferença para os dados aqui encontrados.

Apesar de não ter havido diferença entre os contrastes, pode-se detectar comportamento diferenciado do PRR das mudas quando submetidas às diferentes doses e fontes de N. O comprimento médio das raízes regeneradas em solução hidropônica pode ser observado na Figura 1.

Nota-se que a aplicação crescente de uréia nas mudas de sesbânia apresentou comportamento quadrático para comprimento médio das raízes regeneradas, com ponto de máxima observado aos $196 \mathrm{mg} / \mathrm{kg}$.

Constatou-se que o efeito foi linear tanto com o uso do sulfato como do nitrato de amônio, ocorrendo aumento do comprimento médio das raízes regeneradas à medida que as doses de $\mathrm{N}$ foram aumentadas.

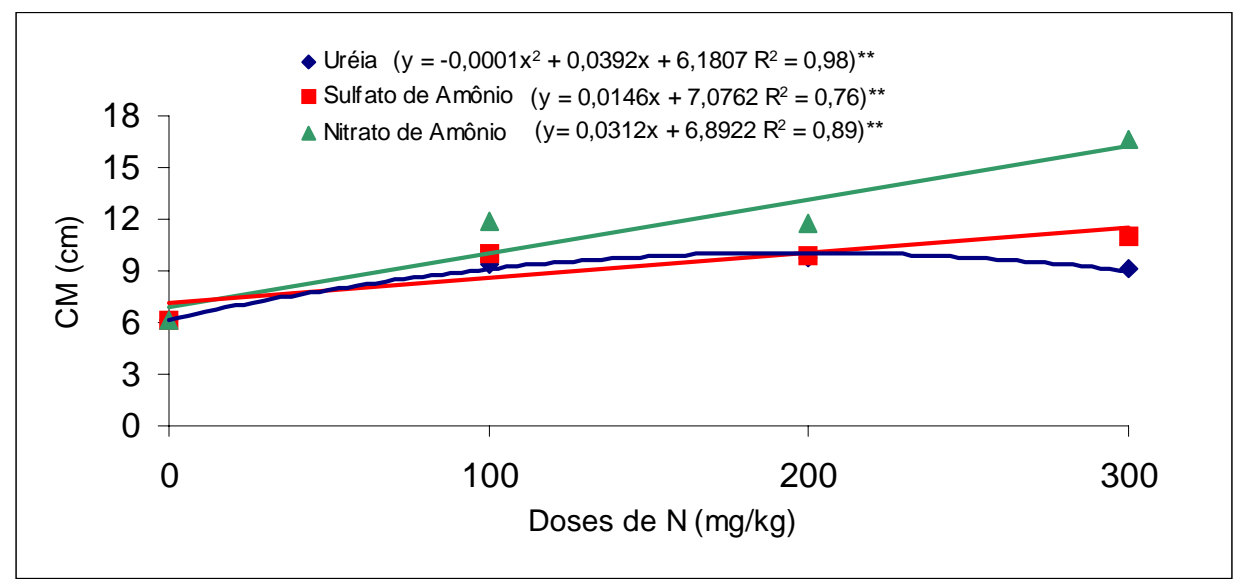

Figura 1 - Comprimento médio das raízes regeneradas das mudas de Sesbania virgata, em solução hidropônica 15 dias após a poda-padrão das raízes.

Figure 1 - Medium length of regenerated roots of Sesbania virgata seedlings in nutrition solution 15 days after standard prunings of the roots. 
As diferenças entre as fontes de $\mathrm{N}$ foram detectadas nos testes de PRR a partir da terceira leitura, realizada nove dias após o transplantio para os tubos (Figuras $2 \mathrm{e}$ 3 ). Estes resultados indicam que pode haver redução do tempo de observação nos experimentos futuros.

Os efeitos das doses de $\mathrm{N}$ no número e no comprimento de raízes regeneradas nos tubos podem ser observados nas Figuras 2 e 3, respectivamente.

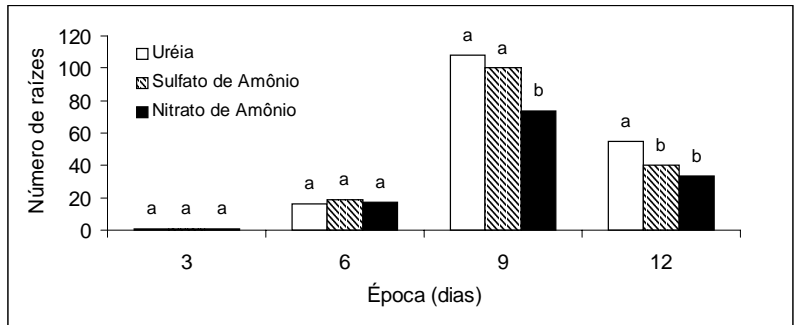

Figura 2 - Número de extremidades de raízes regeneradas das mudas de Sesbania virgata, nos tubos, 12 dias após poda-padrão das raízes. $\mathrm{N}$ : dados transformados em log $(X+10)$.

Figure 2 - Top number of regenerated roots of Sesbania virgata seedlings, in tubes, 12 days after standard pruning of the roots. $\mathrm{N}$ : data transformed into $\log (X+10)$.

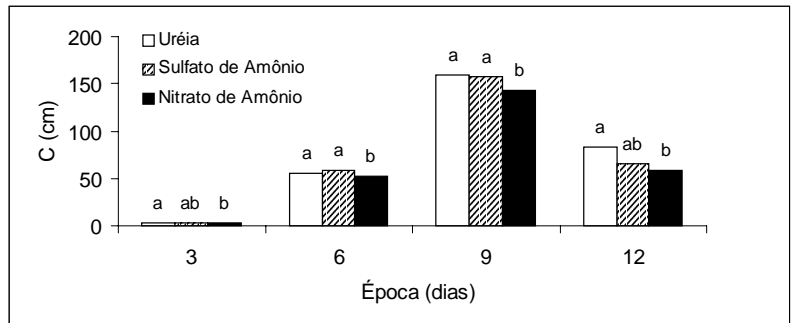

Figura 3 - Comprimento de raízes regeneradas das mudas de Sesbania virgata, nos tubos, 12 dias após poda-padrão das raízes. C: dados transformados em log $(X+10)$.

Figure 3 - Length of regenerated roots of Sesbania virgata seedlings, in tubes, 12 days after standard pruning of the roots. C: data transformed into $\log (X+10)$.

\section{CONCLUSÕES}

A inoculação natural é suficiente para produzir mudas de sesbânia no substrato constituído de bagaço de cana-de-açúcar e torta de filtro de usina açucareira (3:2, v:v), não sendo necessária a inoculação ou a adição de $\mathrm{N}$ em diferentes fontes e doses.

R. Árvore, Viçosa-MG, v.27, n.4, p.443-449, 2003

\section{REFERÊNCIAS BIBLIOGRÁFICAS}

BARROSO, D. G. et al. Efeitos da adubação em mudas de sabiá (Mimosa caesalpiniaefolia Benth) e aroeira (Schinus terebinthifolius Raddi) produzidas em substrato constituído por resíduos agro-industriais. Revista Árvore, v. 22, n.4, p.433-441, 1998

BARROSO, D. G. Qualidade de mudas de Eucalyptus camaldulensis e Eucalyptus urophylla, produzidas em tubetes e em blocos prensados, com diferentes substratos. 1999. 83 f. Tese (Doutorado em Produção Vegetal) Universidade Estadual do Norte Fluminense, Campos dos Goytacazes, 1999.

BARROSO, D. G. et al. Efeito de diferentes doses de nitrogênio em mudas de sesbânia (Sesbânia virgata Raddi) e sabiá (Mimosa caesalpiniifolia Benth), produzidas em resíduos agro-industriais como substrato. In: FOREST 2000, Porto Seguro, 2000. Resumos Técnicos... Porto Seguro: Biosfera, 2000, p. 120-121.

CARnEIRO, J. G. A. Produção e controle de qualidade de mudas florestais. Curitiba: UFPR/FUPEF; Campos: UENF, 1995. $451 \mathrm{p}$.

COSTA JÚNIOR, P. F. Comportamento de leguminosas arbóreas inoculadas com fungos micorrízicos arbusculares e rizóbio em estéril de argila. 1997. 64 f. Dissertação (Mestrado em Produção Vegetal) - Universidade Estadual do Norte Fluminense, Campos dos Goytacazes, 1997.

FARIA, S. M.; MELLO, R. B. Seleção de estirpes de rizóbio para leguminosas arbóreas. In: REUNIÃO BRASILEIRA DE FERTILIDADE DO SOLO E NUTRIÇÃO DE PLANTAS, 23., REUNIÃO BRASILEIRA SOBRE MICORRIZAS, 7., SIMPÓSIO BRASILEIRO DE MICROBIOLOGIA DO SOLO, 5., REUNIÃO BRASILEIRA DE BIOLOGIA DO SOLO, 2., 1998, Caxambu. Anais... Caxambu: 1998. p. 609.

FREIRE, J. R. J. Fixação do nitrogênio pela simbiose rizóbio/leguminosas. In: CARDOSO, E. J. B. N.; TSAI, S.; NEVES, M. C. P. (Coords.). Microbiologia do solo. Campinas: SBCS, 1992. p.121-140.

LELES, P. S. S. Produção de mudas de Eucalyptus camaldulensis, Eucalyptus grandis e Eucalyptus pellita em blocos prensados e em tubetes. 1998. $73 \mathrm{f}$. Tese (Doutorado em Produção Vegetal) - Universidade Estadual do Norte Fluminense, Campos dos Goytacazes, 1998.

MORGADO, I. F. Resíduos agroindustriais prensados como substrato para a produção de mudas de Eucalyptus grandis Hill ex Maiden e Saccharum spp. 1998. 102 f. Tese (Doutorado em Produção Vegetal) - Universidade Estadual do Norte Fluminense, Campos dos Goytacazes, 1998. 
NOVAES, A. B. Avaliação morfofisiológica da qualidade de mudas de Pinus taeda $L$., produzidas em raiz nua e em diferentes tipos de recipientes. 1998. $118 \mathrm{f}$. Tese

(Doutorado em Engenharia Florestal) - Universidade Federal do Paraná, Curitiba, 1998.

SAMÔR, O. J. M. Comportamento de mudas de Sesbania virgata e Anadenanthera macrocarpa, produzidas em diferentes recipientes e substratos, destinadas à recuperação de áreas degradadas pela extração de argila. 1999. $70 \mathrm{f}$.

Dissertação (Mestrado em Produção Vegetal) - Universidade Estadual do Norte Fluminense, Campos dos Goytacazes, 1999.
SANTOS, D. R.; MOREIRA, F. M. S.; SIQUEIRA, J.O. Fósforo, fungos micorrízicos arbusculares e rizóbio no crescimento, nodulação e fixação biológica de nitrogênio em Sesbania virgata e Sesbania rostrata. In: REUNIÃO BRASILEIRA DE FERTILIDADE DO SOLO E NUTRIÇÃO DE PLANTAS, 23., REUNIÃO BRASILEIRA SOBRE MICORRIZAS, 7., SIMPÓSIO BRASILEIRO DE MICROBIOLOGIA DO SOLO, 5., REUNIÃO BRASILEIRA DE BIOLOGIA DO SOLO, 2., 1998, Caxambu. Anais.... Caxambu: 1998. p. 204. 